\title{
Effect of fatty acids on leukocyte function
}

C. Pompéiaํㅡ, L.R. Lopes², C.K. Miyasaka1 ${ }^{1}$ J. Procópio ${ }^{1}$, P. Sannomiya ${ }^{2}$ and R. Curi ${ }^{1}$
Departamentos de ${ }^{1}$ Fisiologia e Biofísica and

2Farmacologia, Instituto de Ciências Biomédicas,

Universidade de São Paulo, São Paulo, SP, Brasil

\section{Correspondence \\ R. Curi \\ Departamento de Fisiologia \\ e Biofísica, ICB, USP \\ Av. Prof. Lineu Prestes, 1524 \\ 05508-900 São Paulo, SP \\ Brasil \\ Fax: + 55-11-818-7285 \\ E-mail: ruicuri@fisio.icb.usp.br \\ Research supported by FAPESP, \\ CNPq, CAPES, The British Council \\ and PRONEX.}

Received January 5, 2000

Accepted June 21, 2000

\section{Abstract}

Fatty acids have various effects on immune and inflammatory responses, acting as intracellular and intercellular mediators. Polyunsaturated fatty acids (PUFAs) of the omega-3 family have overall suppressive effects, inhibiting lymphocyte proliferation, antibody and cytokine production, adhesion molecule expression, natural killer cell activity and triggering cell death. The omega- 6 PUFAs have both inhibitory and stimulatory effects. The most studied of these is arachidonic acid that can be oxidized to eicosanoids, such as prostaglandins, leukotrienes and thromboxanes, all of which are potent mediators of inflammation. Nevertheless, it has been found that many of the effects of PUFA on immune and inflammatory responses are not dependent on eicosanoid generation. Fatty acids have also been found to modulate phagocytosis, reactive oxygen species production, cytokine production and leukocyte migration, also interfering with antigen presentation by macrophages. The importance of fatty acids in immune function has been corroborated by many clinical trials in which patients show improvement when submitted to fatty acid supplementation. Several mechanisms have been proposed to explain fatty acid modulation of immune response, such as changes in membrane fluidity and signal transduction pathways, regulation of gene transcription, protein acylation, and calcium release. In this review, evidence is presented to support the proposition that changes in cell metabolism also play an important role in the effect of fatty acids on leukocyte functioning, as fatty acids regulate glucose and glutamine metabolism and mitochondrial depolarization.

\section{Introduction}

Fats are usually thought of as dispensable or even harmful components of foodstuffs, being associated with atherosclerosis and obesity. Doctors and mothers discourage their consumption by children because of their low nutritional value and because they might displace other high quality nutrients, such as

\author{
Key words \\ - Fatty acids \\ - Immune system \\ - Inflammation \\ - Dietary lipids \\ - Leukocytes
}


oil-soluble vitamins, isoprenols - and whichever other substance that can be extracted by organic solvents, which is the definition of a lipid. Among these are the fatty acids, carboxylic acids to the organic chemist, which vary from 2 to 26 carbons, with none, one or various unsaturations. Fatty acids have dissociation constants ( $\mathrm{pKa}$ ) of about 4.5 , which means that, at physiological $\mathrm{pH} 7.4$, the vast majority (about 1000:1) of these molecules will be in the dissociated form (as a salt). The acid and salt forms of fatty acids, for instance, butyric acid and butyrate, arachidonic acid and arachidonate, will be considered here as interchangeable chemical forms, as accepted by most investigators.

It has been known since the nineteenth century that fatty acids can be synthesized from other nutrients, such as carbohydrates and proteins. But even among these simple molecules there are some that are essential to the organism, i.e., the generally known polyunsaturated fatty acids (PUFAs). Notwithstanding their simple nature, these molecules generate a series of highly specialized and potent cell mediators - such as the prostaglandins (PG) and leukotrienes (LT). Fatty acids are also important components of other inter- or intracellular communication molecules, such as platelet activating factor, diacylglycerol and ceramides. As this review will try to point out, even the least sophisticated fatty acids such as the volatile fatty acids or the long-chain saturated fatty acids have important roles in cell metabolism, structure and regulation, with considerable implications in the immune function when the cells in question are leukocytes.

The immune system works with specific and nonspecific recognition of foreign molecules, leading to their inactivation or destruction also by specific or nonspecific means. The specific immune response and the generalized inflammatory reactions are tightly interwoven. To understand the relation between fatty acids and immunology it is therefore necessary to consider their role in inflammation as well as their effect on leukocytes. Fatty acids interfere with many steps of inflammation, such as vascular contraction, chemotaxis, cell adhesion and diapedesis, and cell activation. Fatty acids can modulate leukocyte function, controlling proliferation, production of cytokines and adhesion molecules, and causing cell death. This review will also point out the changes in cell metabolism as an additional mechanism for the modulation of leukocyte function by fatty acids. Many of the effects just mentioned occur via eicosanoids - PG, LT, thromboxanes (TX) and lipoxins - derived from arachidonic acid. This review will focus on the effect of free fatty acids and will not particularly explore eicosanoid actions, frequently mentioned in pharmacology textbooks.

\section{Effects of fatty acids on inflammation}

Inflammation comprises a series of events associated with tissue injury caused by nonspecific agents or by immune-mediated reactions. The typical description of inflammation involves 4 main features: swelling, pain, increases of temperature, and erythema. Fatty acids not only can cause inflammation, particularly arachidonic acid, but also mediate many of its effects.

\section{Regulation of vascular permeability and contraction, pain and fever}

Arachidonic acid lowers the pain threshold, although it cannot cause pain itself. This hyperalgesic action can be reproduced experimentally, although in a less efficient way, by linoleic, linolenic and gamma-linolenic acids. Although the inhibition of cyclooxygenase is important for the effect of arachidonic acid, it has no action on the mild hyperalgesia brought about by other PUFAs. Prostaglandins are also important mediators in the induction of fever by their action on the central nervous system. On the other 
hand, it has been found that diets rich in omega- 3 fatty acids prevent fever caused by lipopolysaccharide (1).

\section{Chemotaxis, adhesion and diapedesis}

Many eicosanoids such as $\mathrm{LTB}_{4}$ and TX have a chemotactic effect. On the other hand, $\mathrm{PGE}_{2}$ can inhibit leukocyte migration and activity. Fatty acids and fatty acid-derived mediators can also regulate the expression of adhesion proteins, such as integrins and selectins, both in leukocytes and in endothelial cells (2). This type of regulation is important for cell rolling, migration, diapedesis and activation.

Seljeflot et al. (2) have shown that endothelial cells of smokers subjected to an omega-3 fatty acid-rich diet express higher levels of E-selectin and vascular cell adhesion molecule-1 (VCAM-1). This effect is opposed by the finding that omega-3 fatty acids and oleate reduce the expression of VCAM-1 (both protein and mRNA levels), E-selectin and intercellular adhesion molecule-1 (ICAM-1) by endothelial cells. Endothelial cells treated with arachidonic acid (20:4 n-6) or its hydroperoxy (HPETE) or hydroxy (HETE) derivatives, have a reduced ability to adhere to neutrophils and monocytes, probably due to the concomitant decrease in the expression of ICAM-1, Eselectin and VCAM-1, quantified both at the protein and mRNA levels (3).

Yaqoob (4) has shown that monounsaturated fatty acid-rich diets lower peripheral monocyte adhesion, whereas Calder's group (3) found that saturated fatty acids enhance cultured macrophage adhesion when compared to PUFA. It has been found that monocytes treated with eicosapentaenoic acid (EPA) have lower expression of ICAM-1, an effect that is opposite to that of docosahexaenoic acid (DHA) (5). After monocyte stimulation, it has been found that both EPA and DHA (omega-3 fatty acids) had a reduced expression of ICAM-1 (5). Young et al. (6) have shown that linoleic acid, a PUFA, increases ICAM-1. Adhesion molecules, such as $\mathrm{CD} 11 \mathrm{~b} / \mathrm{CD} 18 / \mathrm{Mac}-1$, a beta2 integrin, are quickly accumulated on the neutrophil surface, whose affinity state is increased upon cell treatment with oleic acid (7). Such oleic acid-treated neutrophils can also aggregate easier and show higher adhesion to endothelial cells (8).

Butyrate, a short-chain fatty acid, has been shown to decrease the expression of ICAM-1 and LFA-3 and to increase constitutive as well as cytokine-induced expression of B7-2 (9).

Omega-3 PUFA can suppress neutrophil chemotactic responsiveness to $\mathrm{LTB}_{4}$ and FMLP. Such effect correlates with inhibition in signal transduction pathways between the receptor and phospholipase $\mathrm{C}$, with suppression of $\mathrm{IP}_{3}$ formation (3). Fish oil-rich diets (rich in omega-3 fatty acids) raise bronchoalveolar fluid myeloperoxidase activity in endotoxin-injected rats (10). This enzyme is associated with oxidative stress since it catalyzes the production of free radicals and is a marker of polymorphonuclear accumulation at the inflammatory site. Short-chain fatty acids, on the other hand, inhibit human polymorphonuclear migration (11).

\section{Phagocyte activation, phagocytosis, respiratory burst (reactive oxygen and nitrogen species) and microbicidal action}

Neutrophils and macrophages usually must be activated in order to phagocytose particles, release protein and lipid mediators and produce reactive oxygen and nitrogen species. Fatty acids have been implicated in such effects. Neutrophils contain NADPH oxidase, an enzyme that generates superoxide. NADPH oxidase can be directly stimulated by arachidonic acid, leading to free radical production, and can probably stimulate cell microbicidal activity and, if the 
system is not adequately controlled, cause tissue injury (12).

Superoxide can be spontaneously or enzymatically, via superoxide dismutase, converted into hydrogen peroxide, another reactive oxygen intermediate. Diets rich in omega6 or saturated fatty acids are associated with lower superoxide and hydrogen peroxide production by stimulated neutrophils (13), which correlates with diminished glucose-6-phosphate dehydrogenase activity. This enzyme is one of the major generators of NADPH synthesis in the cell, and NADPH, in turn, is an essential substrate for superoxide generation by NADPH oxidase (13). Patients fed diets containing fish oil (omega-3 fatty acids) show decreased production of reactive oxygen species by stimulated neutrophils, which is independent of cyclooxygenase and degranulation (14).

The presence of medium-chain fatty acidcontaining phospholipids in neutrophil culture increases the production of reactive oxygen species, whereas in vivo treatment with these fatty acids reduces hydrogen peroxide production, nitroblue tetrazolium reduction, and impairs phagocytosis and Staphylococcus aureus killing (15).

Another important molecule produced by phagocytes is nitric oxide (NO). The production of NO is catalyzed in phagocytes by inducible NO-synthase (iNOS), upon cell activation. The transcription of iNOS and the production of NO by macrophages can be inhibited by PUFA, the effect being greater for 22-carbon than 20-carbon fatty acids, and for omega- 3 fatty acids, particularly DHA (16).

As mentioned before, the production of reactive oxygen and nitrogen species by phagocytes is important for these cells to exert their microbicidal function. However, upon cell hyperstimulation, such as in chronic inflammatory or immune diseases, reactive species are deleterious to the organism. The general mechanisms for cell protection involve the production of enzymatic (such as catalase, superoxide dismutase, glutathione peroxidase) and non-enzymatic (such as tocopherol, ascorbic acid, glutathione) antioxidants. Diets rich in omega-3 fatty acids increase lipid peroxidation while concomitantly raising glutathione peroxidase activity. This enzyme is particularly important in cell protection from oxidant stress under physiological conditions, converting peroxides into their alcoholic derivatives, at the expense of glutathione (17).

The microbicidal activity of neutrophils also depends on enzymes and other toxic substances present in their granules released upon cell activation. Myeloperoxidase and transferrin, but not beta-glucuronidase release from activated neutrophils is inhibited by cell treatment with unsaturated fatty acids with odd carbon number, such as 13:1, 17:1 and 19:1. Some of these odd-numbered fatty acids increase, while others decrease superoxide generation (18).

PUFAs usually have an inhibitory effect on phagocytosis and phagocyte microbicidal activity when added to diets, but can stimulate these activities when directly added to cell cultures, although some experimental data are contradictory. Macrophages cultured in myristate- or palmitate-enriched medium or long-chain saturated fatty acids show decreased phagocytic activity against zymosan particles, although cells treated with PUFA, especially arachidonic acid and DHA, show an increased phagocytic capacity and microbicidal action. This effect is associated with $\mathrm{PLA}_{2}$ regulation, calcium release and tyrosine kinase activity (19). Neutrophils treated with long-chain fatty acids with at least 3 double bonds, especially arachidonic acid and fatty acids of the omega- 3 subclass, show enhanced antiparasitic activity against Plasmodium falciparum (20). The addition of cyclooxygenase and lipoxygenase inhibitors has no effect on the increased cytotoxicity induced by the fatty acids, which implies that eicosanoids do not seem to mediate such cell modulation (20). Macrophages from rats 
fed PUFAs showed decreased phagocytic capacity, as well as lowered cell migration compared to those from saturated fatty acidfed animals, which correlated with a decrease in the pentose-phosphate pathway and glutaminase activities (21). Short-chain fatty acids strongly inhibit Staphylococcus aureus phagocytosis and killing by human phagocytes (11).

Macrophages not only kill microorganisms but also tumor cells. The cultivation of macrophages in the presence of DHA (22:6 $n-3)$ led to decreased lysis of mastocytoma cells, an effect that was partially reproduced by arachidonic acid, potentiated by IFNgamma and independent of cyclooxygenase products and bacterial phagocytic action (3).

\section{Production of cytokines and other cell mediators, antigen presentation and lymphocyte activation}

Activated leukocytic inflammatory cells produce many cytokines important in both inflammatory and immune reactions. Gamma-linolenic acid reduces IL-1 beta secretion by monocytes $(22)$. Other authors $(23,24)$ have found that omega-6 PUFAs generally raise IL-1 production and that omega-3 PUFAs have the opposite effect.

The data on the effects of PUFA on TNFalpha production by phagocytes are contradictory. Gamma-linolenic and arachidonic acids (omega-6) administered both in vivo and in vitro can lower TNF-alpha production by monocytes or macrophages and the effect of the latter is reversed by treatment with indomethacin (22), indicating that PG or TX are involved in TNF production. Chavali et al. (25) showed that TNF-alpha levels can be raised by diets rich in omega-3 fatty acids, whereas Kelley et al. (23) showed decreased TNF-alpha secretion by neutrophils from DHA-fed subjects. As to the volatile fatty acids, the delivery of butyrate via liposomes or its addition to culture medium decreases TNF-alpha production by Kupffer cells (26).
On the other hand, human neutrophils treated with omega-3 fatty acids show decreased production of IFN-gamma (24).

Linoleic acid, an omega-6 PUFA, increases the expression of IL-8, a proinflammatory chemokine produced by monocytes, fibroblasts and endothelial cells (6). Omega6 fatty acid-rich diets and direct incubation of macrophages with arachidonic acid also raise IL-6 concentration, possibly via generation of $\mathrm{PGI}_{2}$ (27). In contrast, IL-8, IL-6 and IL-10 expression is lowered by omega- 3 fatty acids and oleate in endothelial cells and monocytes, an effect associated with reduced adhesion molecule expression and lower adhesion capacity of monocytes $(25,27)$.

Monocytes from animals fed diets rich in omega-3 fatty acids (fish oil) have lower antigen-presenting capability (5), that may be associated with a decreased expression of major histocompatibility complex II (MHC II) molecules on macrophages, which are essential for antigen presentation to lymphocytes (5). PUFAs, especially DHA, can inhibit the expression of these molecules in INF-gamma-stimulated cells. It was shown that this effect on MHC II expression is necessary for antigen presentation by macrophages, independent of cytotoxicity and inhibition of cyclooxygenase and is detectable at the mRNA level (28). Butyrate, a volatile fatty acid, reduces macrophage ability to stimulate lymphocytes $(9,26)$.

Diets rich in omega-3 fatty acids have lower levels of $\mathrm{PGE}_{2}, 5$-keto-PGF1 alpha and $\mathrm{TXB}_{2}$ compared to those rich in omega- 6 fatty acids (25). Omega-3 fatty acid-rich diets can also decrease $\mathrm{LTB}_{4}$ production, which is correlated with lower neutrophil chemotaxis. On the other hand, as expected, diets rich in omega- 6 fatty acids increase $\mathrm{PGE}_{2}$, $\mathrm{LTB}_{4}, \mathrm{LTC}_{4}, \mathrm{LTD}_{4}, 6$-keto-PGF1alpha and $\mathrm{TXB}_{2}$, probably due to greater production of arachidonic acid, the eicosanoid precursor. Butyrate can increase Kupffer cell production of $\mathrm{PGE}_{2}$ and upregulate inducible cyclooxygenase mRNA levels (26). 
Another lipid mediator produced by phagocytes that is important in inflammation and in the immune response is the platelet-activating factor. The production of this mediator in stimulated neutrophils is decreased in alphalinoleate-rich diets (omega-3) when compared with linoleate and is associated with decreased availability of lyso-platelet activating factor, its precursor, probably due to inhibition in transacylase activity (29).

Fatty acid-rich diets, particularly of the omega-3 type, are frequently associated with diminished hypersensitivity reactions. Part of this effect may be due to lowered histamine production or release. Thus, omega-3 PUFAs reduce histamine content in the supernatant of mast cell culture, whereas arachidonic acid and short-chain fatty acids have an opposite effect (30). Moreover, omega-3 PUFA also lowered histamine release upon cell stimulation with a ionophore (30). On the other hand, arachidonic acid increases the intracellular histamine content, while omega-3 fatty acids decrease it (30).

\section{Effects of fatty acids on lymphocytes}

\section{Cell activation}

Lymphocyte activation is usually inhibited by fatty acids, particularly by PUFA and volatile fatty acids. Thus, the pretreatment of animals with butyrate was shown to inhibit T-cell proliferation to immobilized anti-CD3 monoclonal antibody (9). Omega-3 fatty acidrich diets are associated with a lower percentage of activated $\mathrm{T}$ and $\mathrm{B}$ cells upon stimulation (14), but with an increased proliferative response to T-cell mitogens (31).

\section{Control of cell proliferation and cell death}

The efficiency of the immune system not only relies on the possibility of specific recognition of foreign antigens, but also on the great amplification of the response by quick induction of lymphocyte proliferation. Many experiments performed with concanavalin A- or lipopolysaccharide-activated lymphocytes show that fatty acids, particularly PUFA and volatile fatty acids, can inhibit cell proliferation by an eicosanoid-independent action $(32,33)$. It is also known that higher doses of these fatty acids can cause cell death via apoptosis or, depending on their concentration, necrosis. Because of these effects, patients with autoimmune diseases or chronic inflammation have been advised to eat PUFA-rich diets. It should be mentioned that a minimum amount of fatty acids is needed for optimum lymphocyte proliferation, as in the absence of fatty acids cells have impaired growth $(34,35)$. Accordingly, it has been shown that lymphocyte DNA synthesis is stimulated by low and inhibited by high free fatty acid concentrations (36).

The cytotoxic effect of PUFA on lymphocytes or leukemia cell lines has long been known. Cell death occurs by apoptosis at concentrations close to the physiological free fatty acid concentrations, as assessed by induction of internucleosomal DNA cleavage, chromatin condensation and nuclear breakdown $(37,38)$. Higher doses of fatty acids preferentially cause necrosis, with a rapid loss of membrane integrity, lysosomal enzyme leakage and cell swelling. Both apoptosis and necrosis seem to be associated with oxidative stress, as they can be partially prevented by antioxidants such as tocopherol (37). As to eicosanoids, they have been found to play an important role in apoptosis depending on the cell type or treatment. In fact, cyclooxygenase inhibitors and sometimes lipoxygenase inhibitors have been found to protect cells from PUFA-induced cytotoxicity (38).

Another class of fatty acids known to be cytotoxic and to have inhibitory effects on lymphocyte proliferation is that of the volatile fatty acids, particularly butyrate. This acid has been shown to suppress T-cell proliferation to immobilized anti-CD3 monoclonal antibody (9). Butyrate-induced cyto- 
toxicity mainly occurs by induction of apoptosis, an effect probably associated with histone acetylation (39). Tumor cells seem to be more susceptible to butyric acid than their normal counterparts. This effect of butyrate is potentiated by propionate and acetate, which might indicate a potential use of this fatty acid in cancer treatment (40).

\section{Antibody production}

Lymphocytes of the B lineage produce specific antibodies in response to antigen stimulation. Antibody production, switching and secretion can be modulated by fatty acids. Thus omega-3 PUFAs inhibit IgA, IgM and IgG production by rat lymphocytes, an effect that can be counteracted by lectin (3). Diets low in fat, deficient in essential fatty acids or rich in fish omega-3 fatty acids are associated with better prognosis and higher survival in antibody-mediated autoimmune diseases and lower IgE production. Omega-3 fatty acids administered orally modulate hypersensitivity reactions (41). Mice fed linoleate (omega-6) have lower IgE production than those fed alpha-linoleate (omega-3). Nevertheless, mice fed alphalinoleate survive more than those fed linoleate after antigen-induced anaphylactic shock, probably because of suppression of eicosanoid synthesis (42). Moreover, mice fed high PUFA-fat diets show a reduced delayed hypersensitivity response to challenge, coupled to a transient decrease in IgM antibody formation, but no alterations in IgG or IgE production (43).

Butyrate, a short-chain fatty acid, enhances IL-6, IL-4 and phorbol 12-myristate 13-acetate IgM production, an effect associated with histone acetylation (44).

\section{Lymphocyte cytokine production and cell surface molecule expression}

As is the case for macrophages and neutrophils, fatty acids can also modulate lym- phocyte cytokine production. Thus, dihomogamma-linoleic acid and arachidonic acid inhibit the production of IL-2 by lymphocytes, an effect that cannot be abolished by cyclooxygenase inhibitors. Most PUFAs can decrease IL-2 production, particularly of the omega-3 class, and EPA has the highest potency among them, an effect associated with their inhibitory action on lymphocyte proliferation and with lowered expression of IL-2 receptor, with a decrease in naive $\mathrm{T}$ cells and a rise in memory $\mathrm{T}$ cells $(3,24)$.

Interleukin-4 production by lymphocytes can be negatively regulated by $\mathrm{PGE}_{2}$ in culture and is found at lower concentrations in animals fed omega-3 fatty acid-rich diets (31).

Palmitic acid enhances the release of IFNgamma by human lymphocytes, while diminishing TNF-alpha production (35). The same authors have shown that other saturated fatty acids are more potent than unsaturated fatty acids in modulating cytokine production, particularly in relation to IFNgamma. Lymphocytes treated with omega-3 fatty acids as well as with butyrate show decreased IFN-gamma production $(24,39)$.

Lymphocyte interaction with other cells, important for immune response regulation and cell migration, depends on the expression of many surface proteins, which can be modulated by fatty acids. Thus, animals fed diets rich in omega-3 fatty acids (fish oil) and omega- 6 fatty acids (olive oil) have decreased expression of LFA-1, ICAM-1 and CD2 $(3,31)$. Pretreatment of lymphocytes with DHA or EPA (omega-3) can reduce the expression of the adhesion molecules Lselectin and LFA-1 (45). The addition of DHA or EPA acids to adhesion assays involving endothelial cells and lymphocytes reduces cell interaction, which is associated with reduced expression of VCAM-1 (45).

Docosahexaenoic acid increases the expression of Thy-1.2 and alters CD8 epitope expression, therefore potentially regulating T-cell activation. There is also regulation of CD44 and CD45 expression by fatty acids, 
particularly oleic acid, although the effect on CD45 can also occur by cell treatment with stearic acid. Oleic acid increases CD44 and CD45 capping, as has been confirmed by in vivo and in vitro studies (46).

Fish oil-rich diets (omega-3 PUFA) are associated with higher TGF-betal expression in $\mathrm{T}$ cells (31). This growth factor is implicated in immune response modulation.

\section{Natural killer and lymphokine- activated killer cell activity}

A very important cell type that acts in natural immunity is the natural killer (NK) cell, which can identify and kill virus-infected or microbe-infected cells, as well as tumor cells. Regarding natural immunity against tumor cells, lymphokine-activated killer (LAK) cells, that efficiently recognize and kill transformed cells, are also very important. As a rule, omega-3 PUFA can inhibit NK cell activity. For instance, high fat diets, particularly those rich in omega-3 PUFAs, can lower NK and LAK cell activity, as well as the number of circulating cells $(14,23)$. This inhibitory effect is also found in other experimental models, such as animals submitted to intravenous infusion with omega-3 PUFA and human cells treated in culture with omega-3 PUFA, that not only suppress NK, but also LAK cell activity (24). Oleic and palmitic acid-rich diets also seem to inhibit NK cell activity (4), whereas short-chain fatty acids, when delivered with parenteral nutrition, increase the cytotoxic activity of these cells (47).

The immune response to tumors depends on the balance of T-helper and T-suppressor cells. It has been found that such a ratio is increased in patients with solid tumors submitted to diets rich in omega-3 PUFA, mainly due to a decrease in suppressor $\mathrm{T}$ cells, indicating that these fatty acids could inhibit tumor growth (48).

Table 1 summarizes the effects of fatty acids on leukocyte function.

\section{Mechanisms of cell modulation induced by fatty acids}

Several groups have investigated the changes induced by fatty acids in cell membrane functioning, transduction pathways, protein acylation, and calcium release in leukocytes (for reviews, see Refs. 3,4). Fatty acids, particularly arachidonic and oleic acids, can also trigger lipid body formation in leukocytes, which seems to play a role during the inflammatory response (49).

Cell functioning, however, depends on the efficient activation of metabolic pathways in order to obtain ATP, and structural molecules such as nucleotides and phospholipids and NADPH for macromolecule synthesis, among others (50). In the absence of glucose and glutamine, leukocytes cannot synthesize macromolecules, migrate or proliferate. The study of cell metabolic regulation is therefore a fundamental approach in immunology and in the study of inflammation. Fatty acids are associated with a series of metabolic pathways, being synthesized from amino acids and glucose, esterified to glycerol to form phospholipids and triacylglycerol or broken down to acetyl CoA or $\mathrm{CO}_{2}$, generating energy. Probably because of this key position in cell metabolic pathways, fatty acids can regulate their own synthesis and breakdown, as well as interfere with neighboring metabolic routes.

\section{Fatty acids, biochemical pathways and mitochondria}

As mentioned before, lipids are important membrane components, sources of energy and of cell mediators, being essential for cell survival and function. There are many feedback loops that guarantee controlled fatty acid metabolism. For instance, animals fed PUFA-rich diets have reduced transcription of many lipogenic enzymes, such as fatty acid synthase, acetyl CoA carboxylase, malic enzyme, pyruvate kinase and 
phosphoenolpyruvate carboxykinase (51). Lymphocytes treated with propionate have impaired lipid synthesis and diminished proliferation and macrophages treated with this fatty acid show changes in metabolism (32, 52).

Many effects of fatty acids on cells can be explained by the uncoupling of mitochondria. Most studies on the uncoupling effect have been conducted on liver, muscle or adipose tissue mitochondria, but the phenomenon has also been characterized in intact lymphocytes (53). Until now, nevertheless, the exact mechanism is not known, although there is a consensus about the interaction between fatty acids and the ADP/ATP antiporter/carrier, which might act as an uncoupling protein (54). Arachidonic acid has been shown to inhibit complexes I and III of the respiratory chain, a fact that might explain its uncoupling effect (55). Such inhibition is associated with a high production of hydrogen peroxide when pyruvate plus malate or succinate are used as substrates. However, some authors believe that, under physiological conditions, fatty acids act only as mild uncouplers, preventing pronounced changes in transmembrane potential that could lead to reactive oxygen species forma- tion (56). The uncoupling effect of fatty acids is greater for PUFAs and considerably mild for short-chain fatty acids (55).

Depolarization of the inner mitochondrial membrane by fatty acids is important because of its consequences for energy metabolism and cell death. As mentioned before, fatty acids, particularly PUFAs, are cytotoxic, causing cell death via apoptosis or necrosis. It has been proposed that these effects can be triggered in the mitochondria. Depolarization is associated with the leakage of cytochrome $\mathrm{c}$, which then can bind to Apaf-1 and trigger the caspase pathway of apoptosis. Concomitantly, depolarization causes impaired ATP synthesis and greater reactive species formation, which can then impair cellular homeostasis and promote lipid peroxidation, respectively. As a consequence, cytoplasmic and lysosomal membrane integrity may be lost, characterizing necrosis. The death pathway which cells will undergo depends, therefore, on the extent of mitochondrial damage triggered by fatty acids (57).

It was once generally considered that lymphocytes, macrophages and neutrophils obtain most of their energy by metabolism and oxidation of glucose and that lympho-

Table 1 - Summary of the effects of fatty acids on leukocytes.

Macrophages and neutrophils

- Fatty acid-fed animals show decreased cell activation, production of reactive oxygen species

(ROS), phagocytosis and antigen presentation (by macrophages) $(5,13-15,19,21,28)$.

- The addition of fatty acids to the cell medium increases ROS formation and phagocytosis $(12,15,20)$.

- Omega-3-rich diets decrease IL-1beta, IL-6 and IL-8 production, whereas omega-6-rich diets increase the production of these cytokines $(6,22-25,27)$.

Lymphocytes

- PUFAs at low concentrations are needed for cell proliferation, but at high concentrations, inhibit proliferation $(14,31-34,36)$.

- PUFAs inhibit antibody synthesis and IL-2 production $(3,24,41,43)$.

- Omega-3-rich diets decrease NK and LAK cell activity $(14,23,24)$.

- Omega-3 fatty acids decrease adhesion molecule expression and lymphocyte adhesion to the endothelium $(3,31,45)$.

General effects

- PUFAs and volatile fatty acids at high concentrations cause cell death via apoptosis or necrosis (37-40).

- Omega-3-rich diets inhibit the production of eicosanoids (PG, LT, TX), whereas omega-6-rich diets have the opposite effect (25). 
cytes which had not been subjected to an immune response (resting lymphocytes) were metabolically quiescent. Evidence has been obtained, however, that the rate of glutamine utilization by these cells is either similar to or greater than that of glucose. Neither glutamine nor glucose are fully oxidized; almost all of the glucose used is converted to lactate and almost all of the glutamine to glutamate, lactate and aspartate (56). On the basis of end the products of metabolism and maximal activities of some enzymes, it has been assumed that a similar if not identical biochemical pathway exists in macrophages, lymphocytes and neutrophils.

The role of high rates of glutaminolysis and glycolysis in lymphocytes, macrophages, and neutrophils has been established. Both processes provide ATP and metabolic intermediates for biosynthetic pathways: glycolysis provides glucose-6-phosphate for the formation of ribose-5-phosphate for DNA and RNA synthesis, NADPH for macromolecule synthesis and glycerol-3-phosphate for phospholipid synthesis, and glutaminolysis provides ammonia and aspartate for purines and pyrimidines for DNA and RNA synthesis (50).

The question that then arises is how it is possible to obtain information on the metabolic regulation of cells and tissues. One means of obtaining this information is by the determination of enzyme activities of metabolic pathways. Key enzymes can provide information on the maximal capacities of certain pathways in metabolism. The quantitative value of the approach depends upon the care with which the enzymes are chosen. They must function only in the pathway being assessed and must catalyze non-equilibrium reactions (50).

Hexokinase is the key enzyme for the control of the flux of metabolites through glycolysis, glucose-6-phosphate dehydrogenase for the pentose-phosphate pathway, citrate synthase for the tricarboxylic acid cycle, and phosphate-dependent glutaminase for glutaminolysis. The activity of these enzymes in lymphoid organs (thymus, spleen and mesenteric lymph nodes) of rats fed a high omega-6 PUFA diet is markedly changed after 6 weeks and this effect is exacerbated after 14 months (13). The administration of an omega-3 PUFA-enriched diet for 6 weeks increases hexokinase in the spleen and thymus and reduces this enzyme activity in the mesenteric lymph nodes, whereas citrate synthase activity is diminished in the spleen and lymph nodes and enhanced in the thymus (17). This type of diet raises the activity of glucose-6-phosphate dehydrogenase in the three lymphoid organs. It is interesting to point out, however, that when fish oil (rich in omega-3 PUFAs) is given by gavage the findings obtained do not fully reproduce those obtained with the fish oil-enriched diet $(17,58)$. This observation indicates that the route of oil administration is able to cause different effects and this subject does deserve to be carefully investigated.

In macrophages isolated from the intraperitoneal cavity of rats fed an omega- 6 PUFA-rich diet, marked changes in phagocytosis capacity, hydrogen peroxide production and also in the metabolism of glucose and glutamine have been reported (59). In studies carried out in vivo and on isolated lymphocytes (60), the reduced proliferative capacity of mesenteric lymph node-derived lymphocytes from rats fed unsaturated fatty acid-rich diets could well be a consequence of changes in glucose and glutamine metabolism. This effect could be due to gene transcription regulation. In fact, fatty acids can have a direct effect on gene transcription by interaction and activation of specific transcription factors, the peroxisome proliferator activated receptors (PPAR), of the family of the retinoic acid receptors. Such proteins, divided into 4 major classes - alpha, beta, gamma and delta-PPAR, dimerize with retinoic acid and can then recognize specific DNA responsive elements positively or negatively modulating gene transcription. It is by 
Table 2 - Summary of the mechanisms proposed for the effect of fatty acids on leukocytes.

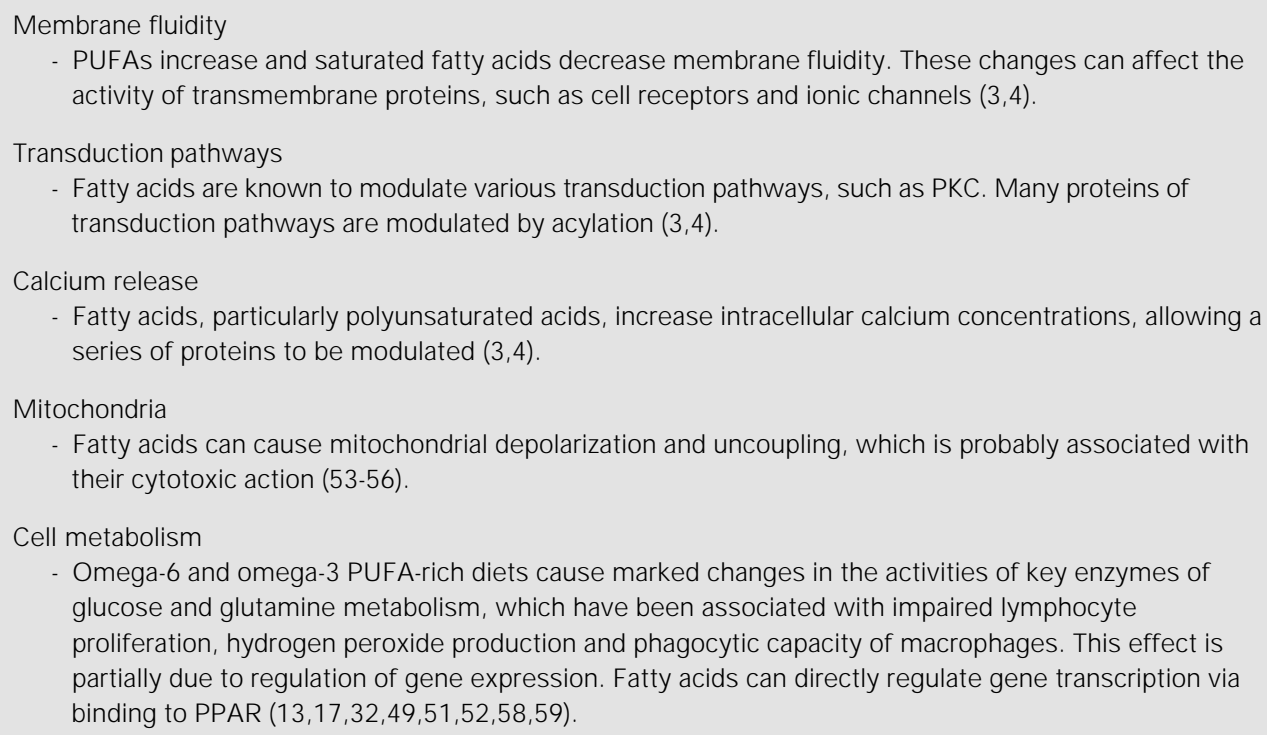

this mechanism that fatty acids regulate their own metabolism and interfere with other metabolic routes, as is the case for linolenic acid induction of acyl-coenzyme A synthetase or oleic acid induction of phosphoenolpyruvate kinase $(49,51)$.

Table 2 summarizes the possible mechanisms of leukocyte modulation by fatty acids.

\section{Concluding remarks}

Experimental evidence points to the important role of fatty acids in the immune and inflammatory responses. Most studies have focused on the effects of PUFAs, particularly omega-3, derived from fish oil. These omega-3 fatty acids generally inhibit immune and inflammatory functions by decreasing lymphocyte proliferation, cytokine production, NK cytotoxicity, and antibody production, among other effects. Many of these studies are based on the effects of fatty acid-rich diets. The fatty acids that most affect the immune system are those of the omega-3 type, which has an inhibitory action, as mentioned before, followed by the omega-6 type, which mostly has a less pro- nounced effect. As to this last group of fatty acids, some of its effects can be attributed to the formation of eicosanoids (PG, TX, LT) or even alteration in glucose and glutamine metabolism. Unfortunately, however, most studies so far have involved mixtures of fatty acids, particularly as diet components. Today, research tends to focus on the separate effect of individual PUFAs, in the search for those with greatest potency in each given condition. Finally, diets rich in medium-chain or long-chain saturated fatty acids have practically no effect on immune and inflammatory functions. On the other hand, shortchain fatty acids, also known as volatile fatty acids, such as butyric acid, have many effects on immune and inflammatory systems, at times of a stimulatory nature and at others of an inhibitory nature. The effects of fatty acid-rich diets are important when considering the treatment of immunosuppressed patients and those with autoimmune conditions. Thus, many studies have been performed on fish oil-fed patients with chronic inflammatory diseases or allergy, usually associated with improvement. Therefore, the importance of fatty acids for the immune and 
inflammatory systems cannot be overlooked. These metabolites are important components of the diet and act as both intracellular and extracellular mediators, positively or negatively regulating physiological and pathological conditions.

\section{References}

1. Kozak W, Soszynski D, Rudolph K, Conn CA \& Kluger MJ (1997). Dietary n-3 fatty acids differentially affect sickness behavior in mice during local and systemic inflammation. American J ournal of Physiology, 272: R1298-R1307.

2. Seljeflot I, Amesen $H$, Brude IR, Nenseter MS, Drevon CA \& Hjermann I (1998). Effects of omega-3 fatty acids and/or antioxidants on endothelial cell markers. European J ournal of Clinical Investigation, 28: 629-635.

3. Calder PC (1998). Immunoregulatory and anti-inflammatory effects of $n-3$ polyunsaturated fatty acids. Brazilian J ournal of Medical and Biological Research, 31: 467490.

4. Yaqoob P (1998). Monounsaturated fats and immune function. Brazilian J ournal of Medical and Biological Research, 31: 453465.

5. Hughes DA \& Pinder AC (1997). n-3 polyunsaturated fatty acids modulate the expression of functionally associated molecules on human monocytes and inhibit antigen-presentation in vitro. Clinical and Experimental Immunology, 110: 516-523.

6. Young VM, Toborek M, Yang F, McClain CJ \& Hennig B (1998). Effect of linoleic acid on endothelial cell inflammatory mediators. Metabolism, 47: 566-572.

7. Sherrington EJ , Sanderson $P \&$ Calder PC (1995). The effect of dietary lipid manipulation on macrophage cell surface molecule expression. Biochemical Society Transactions, 23: 272S.

8. Mastrangelo AM, J eitner TM \& Eaton J W (1998). Oleic acid increases cell surface expression and activity of CD11b on human neutrophils. J ournal of Immunology, 161: 4268-4275.

9. Bohmig GA, Krieger PM, Saemann MD, Wenhardt C, Pohanka E \& Zlabinger GJ (1997). n-butyrate downregulates the stimulatory function of peripheral bloodderived antigen-presenting cells: a potential mechanism for modulating T-cell responses by short-chain fatty acids. Immunology, 92: 234-243.

10. Mancuso P, Whelan J, DeMichele SJ, Snider CC, Guszcza J A \& Karlstad MD (1997). Dietary fish oil and fish and borage oil suppress intrapulmonary proinflamma- tory eicosanoid biosynthesis and attenuate pulmonary neutrophil accumulation in endotoxic rats. Critical Care Medicine, 25: 1198-1206.

11. Touyama M, Kusano N \& Saito A (1995). Effects of the Prevotella intermedia culture filtrate and short-chain fatty acids on human polymorphonuclear neutrophil functions. Kansenshogaku Zasshi. J ournal of the J apanese Association for Infectious Diseases, 69: 1348-1355.

12. Lu DJ \& Grinstein S (1990). ATP and guanine nucleotide dependence of neutrophil activation. J ournal of Biological Chemistry, 265: 13721-13729.

13. Lopes LR, Laurindo FR, Mancini-Filho J, Curi R \& Sannomiya P (1999). NADPHoxidase activity and lipid peroxidation in neutrophils from rats fed fat-rich diets. Cell Biochemistry and Function, 17: 5764.

14. Robinson LE \& Field CJ (1998). Dietary long-chain (n-3) fatty acids facilitate immune cell activation in sedentary, but not exercise-trained rats. J ournal of Nutrition, 128: 498-504.

15. Bellinati-Pires $R$, Waitzberg $\mathrm{DL}$, Salgado MM \& Carneiro-Sampaio MM (1993). Functional alterations of human neutrophils by medium-chain triglyceride emulsions: evaluation of phagocytosis, bacterial killing, and oxidative activity. J ournal of Leukocyte Biology, 53: 404-410.

16. Khair-El-Din T, Sicher SC, Vazquez MA, Chung GW, Stallworth KA, Kitamura K, Miller RT \& Lu CY (1996). Transcription of the murine iNOS gene is inhibited by docosahexaenoic acid, a major constituent of fetal and neonatal sera as well as fish oils. J ournal of Experimental Medicine, 183: 1241-1246.

17. Guimarães AR \& Curi R (1991). Metabolic changes induced by $w-3$ polyunsaturated fatty acid rich-diet ( $w-3$ PUFA) on the thymus, spleen and mesenteric lymph nodes of adult rats. Biochemistry International, 25: 689-695.

18. Ishida-Okawara A, Tsuchiya $T$, Nunoi $H$, Mizuno S \& Suzuki K (1996). Modulation of degranulation and superoxide generation in human neutrophils by unsaturated fatty acids of odd carbon numbers. Biochimica et Biophysica Acta, 1314: 239-
246.

19. Zheng L, Zomerdijk TP, Van Den Barselaar MT, Geertsma MF, Van Furth R \& Nibbering PH (1999). Arachidonic acid, but not its metabolites, is essential for FcgammaR-stimulated intracellular killing of Staphylococcus aureus by human monocytes. Immunology, 96: 90-97.

20. Kumaratilake LM, Ferrante $A$, Robinson BS, J aeger T \& Poulos A (1997). Enhancement of neutrophil-mediated killing of Plasmodium falciparum asexual blood forms by fatty acids: importance of fatty acid structure. Infection and Immunity, 65: 4152-4157.

21. Guimarães AR, Costa Rosa LF, Safi D \& Curi R (1993). Effect of a polyunsaturated fatty acid-rich diet on macrophage and lymphocyte metabolism of diabetic rats. Brazilian J ournal of Medical and Biological Research, 26: 813-818.

22. Deluca P, Rossetti RG, Alavian C, Karim P \& Zurier RB (1999). Effects of gammalinolenic acid on interleukin-1 beta and tumor necrosis factor-alpha secretion by stimulated human peripheral blood monocytes: studies in vitro and in vivo. J ournal of Investigative Medicine, 47: 246-250.

23. Kelley DS, Taylor PC, Nelson GJ , Schmidt PC, Ferretti A, Erickson KL, Yu R, Chandra RK \& Mackey BE (1999). Docosahexaenoic acid ingestion inhibits natural killer cell activity and production of inflammatory mediators in young healthy men. Lipids, 34: 317-324.

24. Purasiri $P$, McKechnie A, Heys SD \& Eremin O (1997). Modulation in vitro of human natural cytotoxicity, lymphocyte proliferative response to mitogens and cytokine production by essential fatty acids. Immunology, 92: 166-172.

25. Chavali SR, Weeks CE, Zhong WW \& Forse RA (1998). Increased production of TNF-alpha and decreased levels of dienoic eicosanoids, IL-6 and IL-10 in mice fed menhaden oil and juniper oil diets in response to an intraperitoneal lethal dose of LPS. Prostaglandins, Leukotrienes and Essential Fatty Acids, 59: 89-93.

26. Perez RV, J ohnson J, Hubbard NE, Erickson K, Morgan M, Kim S, Rudich SM, Katznelson S \& German J B (1998). Selective targeting of Kupffer cells with liposo- 
mal butyrate augments portal venous transfusion-induced immunosuppression. Transplantation, 65: 1294-1298.

27. Hayashi $\mathrm{N}$, Tashiro T, Yamamori H, Takagi $K$, Morishima $Y$, Otsubo $Y$, Sugiura $T$, Furukawa K, Nitta H, Nakajima N, Suzuki $\mathrm{N} \&$ Ito I (1998). Effects of intravenous omega-3 and omega- 6 fat emulsion on cytokine production and delayed type hypersensitivity in burned rats receiving total parenteral nutrition. J ournal of Parenteral and Enteral Nutrition, 22: 363-367.

28. Khair-el-Din TA, Sicher SC, Vazquez MA, Wright WJ \& Lu CY (1995). Docosahexaenoic acid, a major constituent of fetal serum and fish oil diets, inhibits IFN gamma-induced la-expression by murine macrophages in vitro. J ournal of Immunology, 154: 1296-1306.

29. Oh-hashi K, Takahashi T, Watanabe S, Kobayashi T \& Okuyama H (1997). Possible mechanisms for the differential effects of high linoleate safflower oil and high alpha-linolenate perilla oil diets on platelet-activating factor production by rat polymorphonuclear leukocytes. J ournal of Lipid Mediators and Cell Signalling, 17: 207-220.

30. Ishihara K, Murata M, Kaneniwa M, Saito $H$, Shinohara K, Maeda-Yamamoto $M$, Kawasaki K \& Ooizumi T (1998). Effect of tetracosahexaenoic acid on the content and release of histamine, and eicosanoid production in MC/9 mouse mast cell. Lipids, 33: 1107-1114.

31. Fernandes G, Bysani C, Venkatraman JT, Tomar V \& Zhao W (1994). Increased TGFbeta and decreased oncogene expression by omega-3 fatty acids in the spleen delays onset of autoimmune disease in B/W mice. J ournal of Immunology, 152: 59795987.

32. Curi R, Bond J A, Calder PC \& Newsholme EA (1993). Propionate regulates lymphocyte proliferation and metabolism. General Pharmacology, 24: 591-597.

33. Egami MI, Guimarães ARP, Nascimento Curi CM \& Curi R (1993). Effect of fatty acid-rich diets on thymocyte proliferation and thymus involution during growing. Physiology and Behavior, 53: 531-534.

34. Spieker-Polet H \& Polet H (1981). Requirement of a combination of a saturated and an unsaturated free fatty acid and fattyacid carrier protein for in vitro growth of lymphocytes. J ournal of Immunology, 126: 949-954.

35. Anel J N, Gonzalez B, Torres J M, M ishal Z, Uriel J \& Pineiro A (1990). Fatty acid metabolism in human lymphocytes. I. Timecourse changes in fatty acid composition and membrane fluidity during blastic transformation of peripheral blood lymphocytes. Biochimica et Biophysica Acta, 1044: 323-331.

36. Karsten S, Schafer G \& Schauder P (1994). Cytokine production and DNA synthesis by human peripheral lymphocytes in response to palmitic, stearic, oleic, and linoleic acid. J oumal of Cellular Physiology, 161: 15-22.

37. Finstad HS, Myhrstad MC, Heimli $H$, Lomo J, Blomhoff HK, Kolset SO \& Drevon CA (1998). Multiplication and death-type of leukemia cell lines exposed to very long-chain polyunsaturated fatty acids. Leukemia, 12: 921-929.

38. Koller M, Wachtler P, David A, Muhr G \& Konig W (1997). Arachidonic acid induces DNA-fragmentation in human polymorphonuclear neutrophil granulocytes. Inflammation, 21: 463-474.

39. Dangond F \& Gullans SR (1998). Differential expression of human histone deacetylase mRNAs in response to immune cell apoptosis induction by trichostatin $\mathrm{A}$ and butyrate. Biochemical and Biophysical Research Communications, 247: 833-837.

40. Vecchia MG, Carnelos-Filho M, CavaglieriFelipe CR, Curi R \& Newsholme EA (1997). Acetate and propionate potentiate the anti-proliferative effect of butyrate on RBL-2H3 growth. General Pharmacology, 29: 725-728.

41. Miyasaka CK, MendonçaJ R, Silva ZL, DeSouza J AA, Tavares-de-Lima W \& Curi R (1999). Modulation of hypersensitivity reaction by lipids given orally. General Pharmacology, 32: 597-602.

42. Oh-hashi K, Watanabe S, Kobayashi T \& Okuyama H (1997). Reevaluation of the effect of a high alpha-linolenate and a high linoleate diet on antigen-induced antibody and anaphylactic responses in mice. Biological and Pharmaceutical Bulletin, 20: 217-223.

43. Crevel RW, Friend J V, Goodwin BF \& Parish WE (1992). High-fat diets and the immune response of $\mathrm{C} 57 \mathrm{BI}$ mice. British J ournal of Nutrition, 67: 17-26.

44. Kawamoto T, Gohda E, Iji H, Fujiwara M \& Yamamoto I (1998). SKW 6.4 cell differentiation induced by interleukin 6 is stimulated by butyrate. Immunopharmacology, 40: 119-130.

45. Khalfoun B, Thibault G, Bardos $P$ \& Lebranchu $Y$ (1996). Docosahexaenoic and eicosapentaenoic acids inhibit in vitro human lymphocyte-endothelial cell adhesion. Transplantation, 62: 1649-1657.

46. Peck MD, Li Z, Han T, Wang W, Jy W, Ahn YS, Ziboh VA, Chu AJ \& Bourguignon
LY (1996). Fatty acid unsaturation increases expression and capping of murine lymphocyte CD44 and CD45. Nutrition, 12: 616-622.

47. Pratt VC, Tappenden KA, M CBurney MI \& Field CJ (1996). Short-chain fatty acidsupplemented total parenteral nutrition improves nonspecific immunity after intestinal resection in rats. J ournal of Parenteral and Enteral Nutrition, 20: 264-271.

48. Gogos CA, Ginopoulos P, Zoumbos NC Apostolidou E \& Kalfarentzos F (1995). The effect of dietary omega-3 polyunsaturated fatty acids on T-lymphocyte subsets of patients with solid tumors. Cancer Detection and Prevention, 19: 415-417.

49. Weller PF, Bozza PT, Yu W \& Dvorak AM (1999). Hanvard Thorndike cytoplasmic lipid bodies in eosinophils: central roles in eicosanoid generation. International Archives of Allergy and Applied Immunology, 118: 450-452.

50. Curi R, Newsholme P, Pithon-Curi TC, Pires-de-Melo M, Garcia C, Homem-deBittencourt J r PI \& Guimarães ARP (1999). Metabolic fate of glutamine in lymphocytes, macrophages and neutrophils. Brazilian J ournal of Medical and Biological Research, 32: 15-21.

51. Schoonjans $K$, Watanabe $M$, Suzuki $H$, Mahfoudi A, Krey G, Wahli W, Grimaldi P, Staels B, Yamamoto T \& AuwerxJ (1995). Induction of the acyl-coenzyme A synthetase gene by fibrates and fatty acids is mediated by a peroxisome proliferator response element in the $C$ promoter. J ournal of Biological Chemistry, 270: 1926919276.

52. Costa-Rosa LFBP, Curi R, Bond JA, Newsholme P \& Newsholme EA (1995). Propionate modifies lipid biosynthesis in rat peritoneal macrophages. General Pharmacology, 26: 411-416.

53. Arslan P, Corps AN, Hesketh TR, Metcalfe J C \& Pozzan T (1984). cis-Unsaturated fatty acids uncouple mitochondria and stimulate glycolysis in intact lymphocytes. Biochemical J ournal, 217: 419-425.

54. Carlsson C, Hakan-Borg LA \& Welsh N (1999). Sodium palmitate induces partial mitochondrial uncoupling and reactive oxygen species in rat pancreatic islets in vitro. Endocrinology, 140: 3422-3428.

55. Cocco T, Di Paola M, Papa S \& Lorusso M (1999). Arachidonic acid interaction with the mitochondrial electron transport chain promotes reactive oxygen species generation. Free Radical Biology and Medicine, 27: 51-59.

56. Korshunov SS, Korkina OV, Ruuge EK, Skulachev VP \& Starkov AA (1998). Fatty 
acids as natural uncouplers preventing generation of $\mathrm{O}_{2} \cdot{ }^{-}$and $\mathrm{H}_{2} \mathrm{O}_{2}$ by mitochondria in the resting state. FEBS Letters, 435: 215-218.

57. Green DR \& Reed J C (1998). Mitochondria and apoptosis. Science, 281: 13091312.

58. Miyasaka CK, Curi R, Mancini-Filho J \& Lajolo FM (1996). Administration of fish oil by gavage increases the activities of hexokinase, glucose-6-phosphate dehydrogenase and citrate synthase in rat lymphoid organs. General Pharmacology, 27: 991-994.

59. Guimarães AR, Costa Rosa LF, Sitnik RH \& Curi R (1991). Effect of polyunsaturated (PUFA n-6) and saturated fatty acid-rich diets on macrophage metabolism and function. Biochemistry International, 23: 533-543.

60. Otton R, Graziola F, De-Souza JAA, Pithon-Curi TC, Hirata MH \& Curi R (1998). Effect of dietary fat on lymphocyte proliferation and metabolism. Cell Biochemistry and Function, 16: 253-259. 